to reimplant that vessel separately, but only if the tear cannot be repaired by direct suturing reinforced with the stent-graft device.

Finally, covering the left subclavian artery was not associated with any acute malperfusion. Admittedly, the series is small and left subclavian malperfusion can become an issue during follow-up. Delayed revascularization is a simple solution to this problem if needed. Two patients have developed type 2 endoleaks and underwent subsequent carotid to subclavian bypass with embolization at the origin. If a disease-specific device were available, it may be easier to preserve the subclavian artery during this procedure. More important, approximately half of the patients presented with malperfusion, and all were readily managed in the hybrid operating room by this technique that optimizes true lumen flow with or without additional endovascular procedures performed promptly enough to avoid permanent end-organ injury.

This procedure is safe and effective, and we will continue to use commercially available stent-grafts with modification because we believe this operation represents an improvement in care, especially for those patients with malperfusion. Our hope is that a new device, specifically designed for this application, will become available to allow for more widespread adoption.

Eric E. Roselli, $M D$

Department of Thoracic and Cardiovascular Surgery

Cleveland Clinic Heart and Vascular Institute

Cleveland, Ohio

\footnotetext{
Reference

1. Roselli EE, Rafael A, Soltesz EG, Canale L, Lytle BW. Simplified frozen elephant trunk repair for acute DeBakey type I dissection. J Thorac Cardiovasc Surg. 2013;145(Suppl):S197-201.

http://dx.doi.org/10.1016/ j.jtcvs.2013.04.018
}

\section{CLINICAL EVIDENCE FROM RANDOMIZED TRIALS, NETWORK META-ANALYSES, AND CONFLICTS OF INTERESTS \\ To the Editor:}

I thank Pagano and colleagues ${ }^{1}$ for the letter on my comment ${ }^{2}$ about their recent article on a mixed-treatment comparison meta-analysis (also known as "network meta-analysis") of randomized trials comparing aprotinin and other antifibrinolytics in adult cardiac surgery. ${ }^{3}$

I am disappointed with some of the content in their reply ${ }^{1}$ because methodologic issues remained unanswered and unclear. Also, their statements could potentially contribute to confusion. It is also disturbing if we carefully explore their discordant results with a recent network meta-analysis of both randomized and observational evidence by Hutton and colleagues, ${ }^{4}$ wherein the authors concluded that "[a]lthough meta-analyses of randomized controlled trials were largely inconclusive, inclusion of observational data suggest concerns remain about the safety of aprotinin. Tranexamic and epsilon-aminocaproic acid are effective alternatives that may be safer for patients."

I acknowledge randomized trials are the most rigorous way of determining whether a causal relation exists between an outcome and a given drug. Particularly, randomized trials have several important features: random allocation to treatments; patients and investigators remain unaware of which treatment was given until the trial is terminated; all therapeutic interventions are treated identically except for the experimental drug; patients are normally analyzed within the group to which they were allocated (intention-to-treat analysis); and the analyses are primary, focused on estimating the effect size in predefined outcomes. On the same line, it is generally accepted that meta-analyses (and more recently, network metaanalyses) are a useful tool to critically assess the totality of clinical evidence in a research question, but when conducted in a comprehensive and transparent way. It is worthy of note that even all data in a study-level metaanalysis came from randomized trials, the power of randomization is completely lost (the objects of analysis are the trials, not the patients), and the data are reduced to the equivalent of those results derived from observational data.

It is not, however, true to say that small-trial bias does not represent an issue when it comes to comparative trials; it is widely documented in the scientific literature whether small "negative" trials could remain unpublished indefinitely, but most important, how the potential manipulation in small trials (eg, selection of specific patients, selective reporting) could lead to more favorable effect sizes compared with those reported in larger randomized trials.

Finally, I have found some of the language in the reply ${ }^{1}$ to be somewhat unfortunately chosen. Particularly, they wonder whether my employment by a governmental public health agency constitutes a declarable conflict of interest; in this sense, I should declare that the opinions on my comment $^{2}$ are completely my own and do not necessarily represent those of any regulatory body. I acknowledge conflicts of interests are common, but they are a condition not a behavior, and there is nothing wrong with having conflict of interests. No statement will solve the conflict of interests problem. It is interesting to show, however, that although the authors technically do not breach the Journal's formal rules regarding declaration of conflicts of interest, as their own earlier research indicates, some of the authors have received honoraria from aprotinin's manufacturer. ${ }^{5}$

Ferrán Catalá-López,

PharmD, MPH, PhD

Centro Superior de Investigación en Salud Pública (CSISP) 
Valencia, Spain

Division of Pharmacoepidemiology and Pharmacovigilance

Spanish Medicines and Healthcare

Products Agency (AEMPS)

Madrid, Spain

Fundación Instituto de Investigación en Servicios de Salud Valencia, Spain

\section{References}

1. Pagano D, Howell N, Senanayake E, Freemantle N. Reply to the editor. J Thorac Cardiovasc Surg. 2013;145:610-1.

2. Catalá-López F. Safety of aprotinin in adult cardiac surgery: revisiting the validity of a mixed-treatment comparison meta-analysis. J Thorac Cardiovasc Surg. 2013;145:610.

3. Howell N, Senanayake E, Freemantle N, Pagano D. Putting the record straight on aprotinin as safe and effective: results from a mixed treatment metaanalysis of trials of aprotinin. $J$ Thorac Cardiovasc Surg. 2013;145:234-40.

4. Hutton B, Joseph L, Fergusson D, Mazer CD, Shapiro S, Tinmouth A. Risks of harms using antifibrinolytics in cardiac surgery: systematic review and network meta-analysis of randomised and observational studies. BMJ. 2012;345:e5798.

5. Pagano D, Howell NJ, Freemantle $\mathrm{N}$ Cunningham D, Bonser RS, Graham TR, et al Bleeding in cardiac surgery: the use of aprotinin does not affect survival. $J$ Thorac Cardiovasc Surg. 2008; 135:495-502

http://dx.doi.org/10.1016/ j.jtcvs.2013.03.041

\section{Reply to the Editor:}

We are grateful to Dr CatalanaLopez for his continued comments regarding our work.

Although we do not want to prolong unnecessarily this correspondence, a couple of points are worthy of note in his response.

First, the suggestion of including observational evidence in the metaanalysis is flawed, and we found ourselves in this position. As Rosenbaum and Rubin, ${ }^{1}$ the propensity score methods used by the authors can only address confounding when case mix adjustment is possible to the extent that the prescriber's decision to prescribe a treatment is otherwise ignorable. This is clearly not the case in our situation, in which the observational studies describe a substantial latency (unmeasurable bias) associated with the decision to prescribe, which has been incorrectly interpreted as a risk associated with aprotonin. $^{2,3}$ Thus, following $\mathrm{Dr}$ Catalana-Lopez's advice in this situation would actually compound the previous errors of interpretation that have led us to our difficulties with this agent.

Second, Dr Catalana-Lopez is simply incorrect when he suggests that network meta-analyses have the same status as observational studies. Network meta-analyses preserve the original randomization in trials, and provide fully conditional estimates of treatment effect. However, network meta-analyses require an additional assumption (of exchangeability; ie, that subjects share characteristics from the overall population and may, thus, appear in any of the trials). Thus, rather than fully giving up the protection of randomization, we may consider network meta-analyses to be quasi-randomized estimates.

Finally, with regard to potential conflicts of interest, they should be declared upfront and leave the reader to decide of their importance. These authors take conflicts of interest seriously and, as indicated by $\mathrm{Dr}$ Catalana-Lopez, have declared all possible conflicts in accordance with required journal requirements.

Domenico Pagano, MD, FRCS, FESC ${ }^{a}$

Nick Freemantle, $P h D^{b}$

${ }^{a}$ Queen Elizabeth Hospital Birmingham

Birmingham, West Midlands, United Kingdom

${ }^{b}$ University College London London, United Kingdom

\section{References}

1. Rosenbaum PR, Rubin DB. The central role of the propensity score in observational studies for causal effects. Biometrika. 1983;70:41-55.

2. Mangano DT, Tudor JC, Dietzel C. The risk associated with aprotinin in cardiac surgery. $N$ Engl $J$ Med. 2006;354:353-65.
3. Mangano DT, Miao Y, Vuylsteke A, Tudor JC, Juneja R, Filipescu D, et al. Mortality associated with aprotinin during 5 years following coronary artery bypass graft surgery. JAMA. 2007;297:471-9.

http://dx.doi.org/10.1016/ j.jtcvs.2013.04.024

GENDER DISPARITY MAY CONTRIBUTE TO THE QUASI PRESERVATION OF CARDIOPROTECTION BY REMOTE PRECONDITIONING WITH ISOFLURANE BUT NOT PROPOFOL IN CABG

To the Editor:

We read with great interest the article by Kottenberg and colleagues ${ }^{1}$ published online in The Journal of Thoracic and Cardiovascular Surgery on March 1, 2013. They have done a series of impressive translational works to explore the underlying mechanisms of cardioprotection by remote ischemic preconditioning (RIPC) with isoflurane or propofol in patients undergoing coronary artery bypass grafting (CABG). ${ }^{1-3}$ In their fundamental study published in Acta Anaesthesiologica Scandinavica, ${ }^{2}$ they designed 2 independent substudies and found that isoflurane may be more effective in the preservation of cardioprotection by RIPC than propofol. Then, based on 2 consequently separated and independent studies, ${ }^{2,3}$ they believed that differential activation of signal transducer and activator of transcription 5 (STAT 5) phosphorylation may be the contributing factor.

Nevertheless, there remains inconclusive information about this interesting issue when looking into the fundamental study. In that study, ${ }^{2}$ the male proportion may be even within the substudy (RIPC vs control with isoflurane; RIPC vs control with propofol); however, it may be uneven between the 2 substudies (isoflurane vs propofol, $89.7 \%$ vs $75.8 \%$; mean difference, $14 \%$ ).

Accumulating evidence from animal studies has supported genderspecific cardioprotection by ischemic 